\title{
Damage simulation for the lifespan of elastomers
}

\author{
P. Brož \\ Professional Association for Science, Research and Consultancy, \\ Czech Republic
}

\begin{abstract}
The principles of continuum damage mechanics (CDM) are employed to solve the fatigue damage characteristics of elastomers. In so doing, both the damage strain energy release rate and the elastic energy, as a function of strain versions, of a damaged material rendered according to Ogden model, are put forward. Further, the damage evolution low is set up to express the fatigue lifespan in terms of the nominal strain amplitude subject to cyclic loading. Fatigue tests of a carbon rubber were performed to stipulate the fatigue life-dependence on the nominal stress amplitude, and to compare experimental data with the theoretical formula derived for the fatigue life. To get the stress-strain curve, uniaxial tension tests on a carbon-filled natural rubber were realized. For the said substance, material parameters were defined by fitting the experimental stressstrain curve to Ogden constitutive simulation.
\end{abstract}

Keywords: carbon rubber, dissipative mechanism, filled elastomers, fitting, hyperelasticity, state law, strain energy potential.

\section{Introduction}

Continuum Damage Mechanics introduces a scope to express the damage constitutive equations and to extend them to $3 \mathrm{D}$, according to computations of construction. Some essential aspects of both general circumstances and numerical studies going with CDM are included in publications [1-4].

The Lemaitre damage evolution law of a damage rate determined by plasticity adding, which is also improved by the elastic energy density, is able to solve the effects: ductile failure, fatigue, creep and creep-fatigue of metals or polymers $[1,6]$. In the case of materials such as composites, concrete and filled elastomers, 
no plasticity exists for other definite dissipative mechanisms arrived at, and the Lemaitre damage law is worthless.

The piece of research on these mechanisms shows a general form for the thermodynamical potential (Helmholtz specific free energy). It plans an extension of plasticity coupled with damage scope to more general constitutive simulations inclusive of internal sliding and friction.

An elastomer is a polymer with the property of elasticity. The term, derived from elastic polymer, is often used interchangeably with the term rubber, and is preferred when referring to vulcanizates. Each of the monomers that link to form the polymer is usually made of carbon, hydrogen, oxygen and/or silicon. Elastomers are amorphous polymers existing above their glass transition temperature, so that considerable segmental motion possible. At ambient temperature, rubbers are thus relatively soft $(\mathrm{E} \sim 3 \mathrm{MPa})$ and deformable. Their primary uses are for seals, adhesives and molded flexible parts.

Elastomers are a failure and cyclic strain amplitudes much lower than the static failure strain; these characteristics in elastomers are the result of the damage initiation and accumulation occasioned by fatigue loading. When a cyclic load is applied on particle-reinforced elastomers, particle separation from the matrix and cracking in the matrix can occur after a certain number of loading cycles, causing the elastomers to damage at different areas. Damage at different areas can coalesce and form macroscopic cracks leading to the final rupture of elastomers. The extent of damage in materials can be dependent upon the loading cycles and the loading amplitudes. The continuum damage mechanics theory could be practical in the inquiry of the fatigue damage characteristics of elastomers.

Some fatigue damage events such as the nucleation and growth of initial defects (microvoids, microcracks and second phase inclusion) may permit the using CDM to study the fatigue behavior via the analysis of the damage evolution indicative of forming microcracks and voids likewise as heterogeneity microstructures segregation before macrocrack initiation.

The aim is to apply the continuum damage mechanics to evolve a theoretical model to define the fatigue service life under controlled strain amplitudes for elastomers. In the statement of a damage model for the fatigue characteristics of elastomers, a constitutive model is required. While the elastomers may exhibit time-dependent behavior, as a first order approximation most elastomeric materials can be considered to behave hyperelastically. The constitutive law of a hyperelastic material can be described in terms of a strain energy potential, which is a function of strain invariants. The strain energy potential is determined as the strain energy stored in the material per unit reference volume.

In conformity with [5], the CDM is applied to analyze the damage evolution and fatigue characteristics of elastomeric materials. Ogden simulation is used to express the strain energy potential by virtue of nominal deviatoric principal elongations and to build the nominal stress and strain relation for hyperelastic materials $[8,9]$. A damage model is developed to establish the fatigue life formula for elastomeric materials. A carbon filled natural rubber material was 
tested to obtain the relation between the nominal stress and the nominal strain, and also the fatigue life as a function of the nominal strain amplitude.

\section{General simulation of thermodynamics}

The concept for a unified damage model is to express the damage rate to the dissipative mechanism, frequently sliding and friction, and to take into account damage being determined by a cumulative quantity of the internal sliding. This applies apart from others materials to filled elastomers in that a dissipative effect exists owing to internal sliding of the macro-molecular chains and on the black carbon filler particles.

\subsection{Thermodynamic variables}

Characterize $v=\left[\varepsilon^{\pi}, \boldsymbol{a}, q, D\right]$ being internal variables joined with $A=\left[-\boldsymbol{\sigma}^{\pi}, \boldsymbol{x}, Q,-Y\right]$. The physical significance of these thermodynamic variables depends on the type of material and of the physical dissipative mechanisms. The strain $\varepsilon^{\pi}$ due to internal sliding is an internal inelastic strain (equal to the plastic strain $\varepsilon^{p}$ in plasticity) and $Y$ is the strain energy release rate density.

\subsection{State and evolution laws}

The expression for the state potential accepting to couple damage and internal friction has the form

$$
\rho \psi=(1-D) \cdot w_{1}(\boldsymbol{\varepsilon})+g(D) \cdot w_{2}\left(\boldsymbol{\varepsilon}-\boldsymbol{\varepsilon}^{\pi}\right)+w_{s}(q, \boldsymbol{a})
$$

where $w_{1}$ and $w_{2}$ define the strain energy density and $w_{s}$ the stored energy density depending on the scalar variable $q$ and the tensorial variable $\boldsymbol{a}$. The function $g(D)$ is really put as $g(D)=1-D$.

For the state laws we obtain $A=\rho \frac{\partial \psi}{\partial v}$

$$
\begin{aligned}
& \boldsymbol{\sigma}=\rho \frac{\partial \psi}{\partial \boldsymbol{\varepsilon}}=(1-D) \frac{\partial\left(w_{1}+w_{2}\right)}{\partial \boldsymbol{\varepsilon}} \rightarrow \tilde{\boldsymbol{\sigma}}=\frac{\partial\left(w_{1}+w_{2}\right)}{\partial \boldsymbol{\varepsilon}} \\
& \boldsymbol{\sigma}^{\pi}=-\rho \frac{\partial \psi}{\partial \boldsymbol{\varepsilon}^{\pi}}=(1-D) \frac{\partial w_{2}}{\partial \boldsymbol{\varepsilon}} \rightarrow \tilde{\boldsymbol{\sigma}}^{\pi}=\frac{\partial w_{2}}{\partial \boldsymbol{\varepsilon}} \\
& \boldsymbol{x}=\rho \frac{\partial \psi}{\partial \boldsymbol{a}}=\frac{\partial w_{s}}{\partial \boldsymbol{a}} \\
& Q=\rho \frac{\partial \psi}{\partial q}=\frac{\partial w_{s}}{\partial q} \\
& Y=-\rho \frac{\partial \psi}{\partial D}=w_{1}+w_{2}
\end{aligned}
$$


They understandably define the effective stresses $\sigma, \sigma^{\pi}$, such as the elasticity law expressed by virtue of strains and of effective stresses does not depend unambiguously on $D$ (strain equivalence principle).

Consider the non-associated dissipative potential

$$
F=f+F_{x}+F_{D}
$$

where

- $f=\left\|\tilde{\boldsymbol{\sigma}}^{\pi}-\boldsymbol{x}\right\|-Q-\sigma_{s}<0$ defines the reversibility domain, $\|\cdot\|$ is a norm in the stresses and $\sigma_{s}$ is the reversibility limit.

- the functions $F_{x}=\frac{\gamma}{2 C_{x}} \boldsymbol{x}: \boldsymbol{x}$ and $Q=Q(q)$ simulate the internal sliding nonlinearity. $F_{x}$ models nonlinear kinematic hardening for metals (Armstrong-Frederic law), it accounts for strain softening and Mullins effect in elastomers.

- $\quad F_{D}=\frac{S}{(s+1)(1-D)}\left(\frac{Y}{S}\right)^{s+1}$ is the damage potential with $S$ and $s$ being the damage parameters. It results in Lemaitre damage evolution for metals and to its generalization to other materials.

The evolution laws deduce from the dissipative potential via the normality rule $\dot{v}=-\dot{\mu} \frac{\partial F}{\partial A}$ with $\mu$ a Lagrange multiplier introduced by the consistency condition $f=0$ and $\dot{f}=0$ for non-viscous materials or given by a viscosity law for viscous materials. $\dot{\pi}=\dot{\mu} /(1-D)$ is obtained equal to the norm of the inelastic strain. This characterizes the cumulative measure $\pi$ of the internal sliding,

$$
\pi=\int_{0}^{t}\left\|\dot{\varepsilon}^{\pi}\right\| d t
$$

that will recover the accumulated plastic strain as $p=\int_{0}^{t} \sqrt{2 / 3 \dot{\boldsymbol{\varepsilon}}^{p}: \dot{\boldsymbol{\varepsilon}}^{p}} d t$ in plasticity.

The generalized damage evolution law is got in the following form:

$$
\begin{aligned}
\dot{D} & =\left(\frac{Y}{S}\right)^{s} \dot{\pi} \\
D & =D_{c} \rightarrow \quad \text { mesocrack initiation }
\end{aligned}
$$

which is in conformity with damage determined by the main dissipative mechanisms through $\pi$ and where $D_{c}$ is critical damage at mesocrack initiation. 


\section{Continuous damage and fatigue of elastomers}

Elastomers may be implemented being hyperelastic with internal friction coupled with damage. Internal viscosity is an additional dissipative mechanism.

\subsection{Hyperelasticity with internal friction coupled with damage}

In this case, the generalization of Lemaitre damage law to any dissipative event is suitable. It exactly requires to be expressed in terms of the finite strains. The strain $\boldsymbol{E}=1 / 2(\boldsymbol{C}-\mathbf{1})$ is the Green-Lagrange strain tensor, with the dilatation tensor $\boldsymbol{C}=\boldsymbol{F}^{\boldsymbol{T}} . \boldsymbol{F}$ and $\boldsymbol{F}$ being the transformation gradient. The associated stress is the second Piola-Kirchhoff stress tensor $\boldsymbol{S}$. Simulating internal fraction of elastomers needs two internal variables. They are the internal inelastic strain $\boldsymbol{E}^{\boldsymbol{n}}$ (instead of $\varepsilon^{\pi}$ for small strains) associated with the opposite of a stress denoted $\boldsymbol{S}^{\boldsymbol{\pi}}$ and the internal sliding variable $\boldsymbol{a}$ associated with residual micro-stress tensor $\boldsymbol{x}$.

The state potential is rendered in the reference configuration,

$$
\rho_{0} \psi=\frac{1}{2}(1-D)\left[w_{1}(\boldsymbol{E})+w_{2}\left(\boldsymbol{E}-\boldsymbol{E}^{\pi}\right)\right]+\frac{1}{2} C_{x} \boldsymbol{\alpha}: \boldsymbol{\alpha}
$$

with $\rho_{0}$ as the density of the underformed material and where:

- $\quad w_{1}$ is an hyperelastic energy density

- for plainness $w_{2}$ is the first term of Mooney-Rivlin development with inelastic strain $\boldsymbol{E}^{\boldsymbol{\pi}}$ yielding a non-constant derivative,

$$
w_{2}=4 C_{20}\left[\operatorname{trace}\left(\boldsymbol{E}-\boldsymbol{E}^{\pi}\right)\right]^{2}=C_{20}\left(I_{1}-2 \operatorname{trace} \boldsymbol{E}^{\pi}-3\right)^{2}
$$

In case of incompressible materials the stresses are gained being

$$
\begin{aligned}
& \boldsymbol{S}=\left.\rho_{0} \frac{\partial \psi}{\partial \boldsymbol{E}}\right|_{\text {det } \boldsymbol{F}=1}=(1-D) \frac{\partial\left(w_{1}-w_{2}\right)}{\partial \boldsymbol{E}}-P \boldsymbol{C}^{-1} \\
& \boldsymbol{S}^{\pi}=-\rho_{0} \frac{\partial \psi}{\partial \boldsymbol{E}^{\pi}}=(1-D) \frac{\partial w_{2}}{\partial \boldsymbol{E}}
\end{aligned}
$$

with the internal pressure $P$ due to incompressibility. The state laws stipulate the effective stresses $\boldsymbol{S}=\boldsymbol{S} /(1-D), \widetilde{\boldsymbol{S}}^{\pi}=\boldsymbol{S}^{\pi} /(1-D)$, the residual internal stresses $\boldsymbol{x}$ and the energy density release rate $Y=w_{1}+w_{2}$. The reversibility criterion is $f=\left\|\widetilde{\boldsymbol{S}}^{\pi}-\boldsymbol{x}\right\|-\sigma_{s}<0$ and $\sigma_{s}$ is the reversibility limit. The evolution laws are obtained from the normality rule with the definition for $F_{x}$ and $F_{D}$ as formerly, the damage law still related by eq. (5) but with the cumulative measure of the internal sliding $\pi=\int_{0}^{t}\left\|\dot{\boldsymbol{E}}^{\pi}\right\| d t$. 


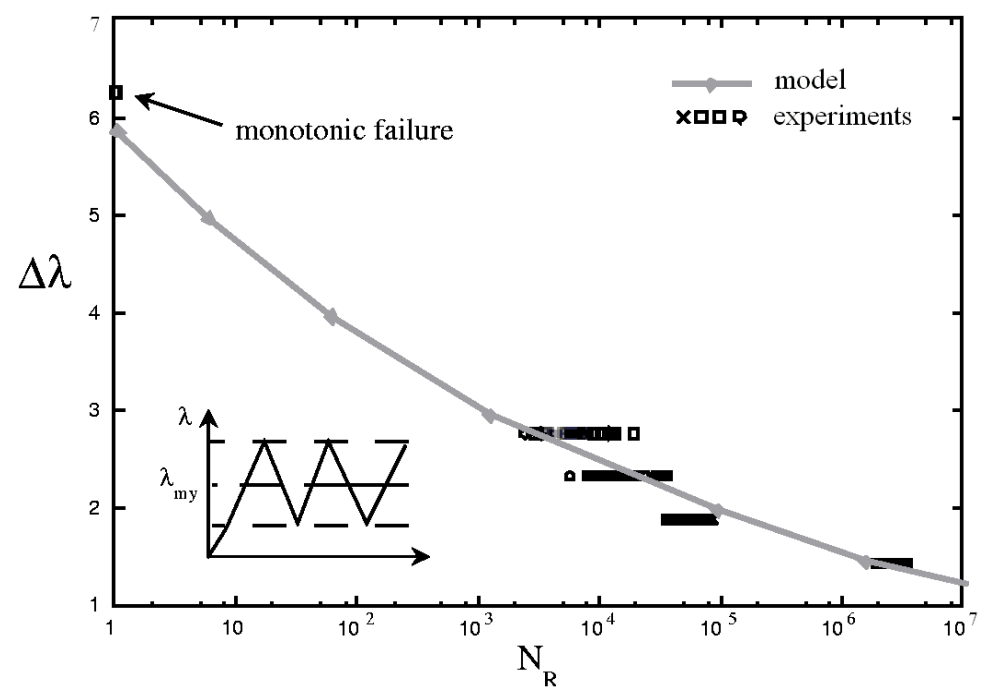

Figure 1: $\quad$ Fatigue curve of a filled SBR for $\lambda_{\text {my }}=2.53$ (taken from [3]).

Observe that with neither damage nor viscosity the model stands for the hysteresis and the stress softening of filled elastomers.

\subsection{Calculation of the fatigue curve}

The hyperelasticity model with internal friction coupled with damage permits to determine the number of cycles to rupture $N_{R}$ in fatigue of elastomers. A periodic elongation $\lambda(t)$ is used (it is larger principal component of $\boldsymbol{F}$ ) with constant amplitude $\Delta \lambda$ and mean elongation $\lambda_{m y}$.

The computed fatigue curve $\Delta \lambda \times N_{R}$ is demonstrated in fig. 1 for a filled Styrene Butadiene Rubber. The measured elongation at rupture in tension $\lambda_{R}=$ 7.2 is also indicated in the diagram.

\section{Ogden model for elastomeric materials}

In Ogden model for incompressible and isotropic elastomer materials, the strain energy potential is described as

$$
U=\sum_{i=1}^{N} \frac{2 \mu_{i}}{\alpha_{i}^{2}}\left(\bar{\lambda}_{1}^{\alpha_{i}}+\bar{\lambda}_{2}^{\alpha_{i}}+\bar{\lambda}_{3}^{\alpha_{i}}-3\right)+\sum_{i=1}^{N} \frac{1}{D_{i}}(J-1)^{2 i}
$$

where $\bar{\lambda}_{i}$ 's are the deviatoric principal elongations and $\bar{\lambda}_{i}=J^{1 / 3} \lambda_{i}$, with $\lambda_{i}$ 's being the principal elongations and $J$ the Jacobian determinant of the deformation gradient; $N$ is the number of terms in the series; and $\mu_{i}{ }^{\prime} \mathrm{s}, \alpha_{i}{ }^{\prime} \mathrm{s}$, and $D_{i}$ 's are temperature-dependent material parameters. The shear modulus $\mu_{0}$ and bulk 
modulus $K_{0}$ for Ogden model at the small strain deformation state are expressed by

$$
\mu_{0}=\sum_{i=1}^{N} \mu_{i}, \quad K_{0}=\frac{2}{D_{1}}
$$

In the uniaxial stress state, the principal elongations $\lambda_{i}$ 's are symbolized by

$$
\lambda_{1}=\lambda_{U}, \quad \lambda_{2}=\lambda_{3}=1 / \sqrt{\lambda_{U}}
$$

where $\lambda_{U}$ is the elongation in the loading direction, $\lambda_{1}$ and $\lambda_{2}$ are the principal elongations on the planes perpendicular to the loading direction.

According to Ogden, the principle of virtual work can be implemented in the following form

$$
T_{U}=\frac{\partial U}{\partial \lambda_{U}}
$$

Let $N=1$ in eqn (9); inserting eqn (11) into eqn (9) results in the strain energy potential for the uniaxial stress state that reads

$$
U=\frac{2 \mu_{1}}{\alpha_{1}^{2}}\left(\lambda_{U}^{\alpha_{1}}+2 \lambda_{U}^{-\alpha_{1} / 2}-3\right)=\frac{2 \mu_{1}}{\alpha_{1}^{2}}\left[\left(1+\varepsilon_{U}\right)^{\alpha_{1}}+2\left(1+\varepsilon_{U}\right)^{-\alpha_{1} / 2}-3\right]
$$

where $\mu_{1}=\mu_{0}=E_{0} / 2(1+v) \approx E_{0} / 3$ for an incompressible material, with $E_{0}$ being Young's modulus at the small strain deformation state. From eqs. (12) and (13), the nominal stress and strain relation under uniaxial tension may be written down being

$$
T_{U}=\frac{2 \mu_{1}}{\alpha_{1}}\left(\lambda_{U}^{\alpha_{1}-1}-\lambda_{U}^{-\left(\alpha_{1} / 2+1\right)}\right)=\frac{2 \mu_{1}}{\alpha_{1}}\left[\left(1+\varepsilon_{U}\right)^{\alpha_{1}-1}-\left(1+\varepsilon_{U}\right)^{-\left(\alpha_{1} / 2+1\right)}\right]
$$

where material parameters $\alpha_{1}$ and $\mu_{1}$ can be found out by fitting experimental nominal stress-strain curve in eqn (14).

\section{The continuum damage mechanics simulation in the uniaxial stress state}

The isotropic damage is supposed concerning the CDM theory, the effective nominal normal stress is expressed as $\bar{T}_{U}=T_{U} /(1-D)$ using the effective nominal stress interpretation via the damage variable $D$. In this way, the nominal stress-strain relation of a damaged material is the same in form as that of an undamaged material in eqn (14) which comes to be 


$$
\bar{T}_{U}=\frac{T_{U}}{1-D}=\frac{2 \mu_{1}}{\alpha_{1}}\left(\lambda_{U}^{\alpha_{1}-1}-\lambda_{U}^{-\left(\alpha_{1} / 2+1\right)}\right)=\frac{2 \mu_{1}}{\alpha_{1}}\left[\left(1+\varepsilon_{U}\right)^{\alpha_{1}-1}-\left(1+\varepsilon_{U}\right)^{-\left(\alpha_{1} / 2+1\right)}\right]
$$

Under a cyclic loading condition, damage accumulates with the number of cycles and the damage evolution depends on the strain amplitude. The time rate change of damage variable $D$ may be represented in terms of the evolution of $D$ with aspect to the number of cycles. Based on this factor, the principal elongation amplitude $\Delta \lambda_{U}$ is used to replace $\lambda_{U}$, and, at that case, the fatigue damage evolution per cycle is related being

$$
\frac{\mathrm{d} D}{\mathrm{~d} N}=\left\{\frac{2 \mu_{1}}{\alpha_{1} S_{0}(1-D)} \times \frac{\left[\lambda_{U}^{\alpha_{1}-1}-\lambda_{U}^{-\left(\alpha_{1} / 2+1\right)}\right]^{2}}{\left[\left(\alpha_{1}-1\right) \lambda_{U}^{\alpha_{1}-2}+\left(\alpha_{1} / 2+1\right) \lambda_{U}^{-\left(\alpha_{1} / 2+2\right)}\right]}\right\}^{s_{0}}
$$

where $\Delta \lambda_{U}$ means the principal elongation amplitude.

Supposing that the damage variable $D$ is zero at the one set of the cyclic loading, that is, $D=0$ when $N=0$, consequently the damage value at any cycle can be stipulated by integrating eqn (15), which yields

$$
\left.\int_{0}^{D} \mathrm{~d}\left[1-(1-D)^{1+s_{0}}\right]=\left(1+s_{0}\right) \int_{0}^{N}\left\{\frac{2 \mu_{1}}{\alpha_{1} S_{0}} \times \frac{\left[\Delta \lambda_{U}^{\alpha_{1}-1}-\Delta \lambda_{U}^{-\left(\alpha_{1} / 2+1\right)}\right]^{2}}{\left[\left(\alpha_{1}-1\right) \Delta \lambda_{U}^{\alpha_{1}-2}+\left(\alpha_{1} / 2+1\right) \Delta \lambda_{U}^{-\left(\alpha_{1} / 2+2\right)}\right.}\right]\right\}^{s_{0}} \mathrm{~d} N
$$

As a result, the relation between the damage variable $D$ and the number of cycles $N$ is

$$
1-(1-D)^{1+s_{0}}=\left(1+s_{0}\right) \times\left\{\frac{2 \mu_{1}}{\alpha_{1} S_{0}} \times \frac{\left[\Delta \lambda_{U}^{\alpha_{1}-1}-\Delta \lambda_{U}^{-\left(\alpha_{1} / 2+1\right)}\right]^{2}}{\left[\left(\alpha_{1}-1\right) \Delta \lambda_{U}^{\alpha_{1}-2}+\left(\alpha_{1} / 2+1\right) \Delta \lambda_{U}^{-\left(\alpha_{1} / 2+2\right)}\right]}\right\}^{s_{0}} N
$$

The condition directly after the fatigue rupture is $D=D_{c}=1$. It should be noted that the stiffness of material is finite immediately before failure, and that is comes to be zero as would be hinted by $D=D_{c}=1$. At the moment of failure, the number of cycles $N=N_{f}$ and the fatigue life $N_{f}$ may be related by

$$
N_{\mathrm{f}}=\left\{\left(1+s_{0}\right)\left\{\frac{2 \mu_{1}}{\alpha_{1} S_{0}} \times \frac{\left[\Delta \lambda_{U}^{\alpha_{1}-1}-\Delta \lambda_{U}^{-\left(\alpha_{1} / 2+1\right)}\right]^{2}}{\left[\left(\alpha_{1}-1\right) \Delta \lambda_{U}^{\alpha_{1}-2}+\left(\alpha_{1} / 2+1\right) \Delta \lambda_{U}^{-\left(\alpha_{1} / 2+2\right)}\right]}\right\}^{s_{0}}\right\}^{-1}
$$

In addition, using $\Delta \lambda_{U}=1+\Delta \varepsilon_{U}$, where $\Delta \varepsilon_{U}$ is the nominal strain amplitude, the formula for fatigue life is rendered being a function of the nominal strain amplitude $\Delta \varepsilon_{U}$, 


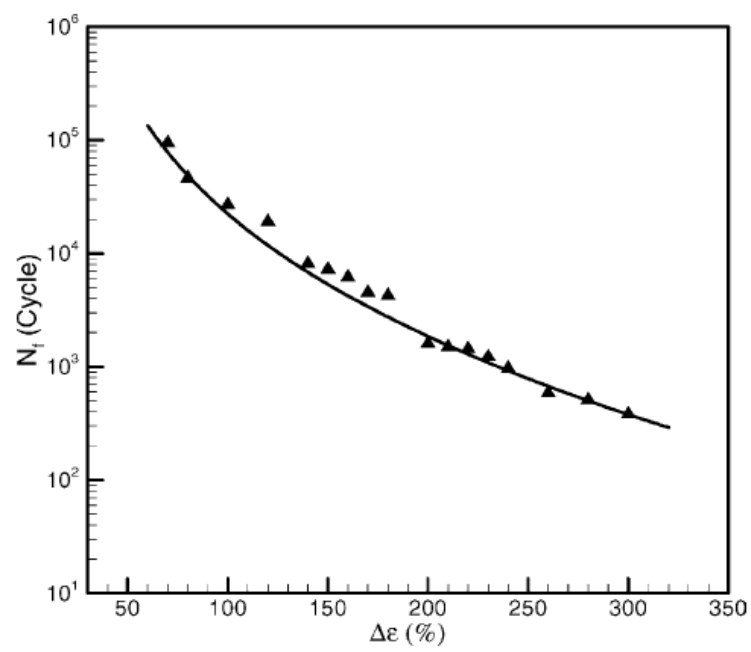

Figure 2: $\quad$ Fatigue life $\mathrm{N}_{\mathrm{f}}$ versus nominal strain amplitude $\Delta \varepsilon$ curve of the rubber material [5].

$$
\left.N_{\mathrm{f}}=\left\{\left(1+s_{0}\right)\left\{\frac{2 \mu_{1}}{\alpha_{1} S_{0}} \times \frac{\left[\left(1+\Delta \varepsilon_{U}\right)^{\alpha_{1}-1}-\left(1+\Delta \varepsilon_{\mathrm{U}}\right)^{-\left(\alpha_{1} / 2+1\right)}\right]^{2}}{\left[\left(\alpha_{1}-1\right)\left(1+\Delta \varepsilon_{U}\right)^{\alpha_{1}-2}+\left(\alpha_{1} / 2+1\right)\left(1+\Delta \varepsilon_{\mathrm{U}}\right)^{-\left(\alpha_{1} / 2+2\right)}\right.}\right]\right\}^{s_{0}}\right\}^{-1}
$$

In eqn (20) $\mu_{1}$ and $\alpha_{1}$ are material parameters same at those in eqn (14) and defined by the experimental nominal stress-strain relation; $s_{0}$ and $S_{0}$ are material parameters determined by the experimental fatigue life as a function of the nominal strain amplitude. Fig. 2 shows the fatigue life as a function of the nominal strain amplitude. The symbols represent experimental data, and the solid line is the curve fitted by eqn (20). Eqn (20) for the carbon-filled natural rubber, is simplified into the form

$$
\left.N_{\mathrm{f}}=2.1 \times 10^{5} \times\left\{\frac{\left[(1+\Delta \varepsilon)^{1.7}-(1+\Delta \varepsilon)^{-2.35}\right]^{2}}{\left[1.7 \times(1+\Delta \varepsilon)^{0.7}+2.35 \times(1+\Delta \varepsilon)^{-3.35}\right.}\right]\right\}^{-2}
$$

\section{Conclusion}

Being a function of the nominal strain amplitude for elastomeric materials, a formula for the fatigue life was presented. Ogden model was used to express the strain energy potential in terms of strain alterations and to build nominal stress and strain relation for elastomeric materials. The theory of continuum damage mechanics was applied to see into damage characteristics for elastomers. A damage model was evolved to derive the formula for the fatigue life in connection with continuum damage mechanics. 
Uniaxial tension experiments on a carbon-filled natural rubber were performed to set the nominal stress and strain curve. Material parameters were found out by fitting the experimental nominal stress-strain curve to Ogden constitutive model for the rubber material. Fatigue tests were also conducted under strain-controlled loading condition at room temperature to determine the fatigue life as a function of the nominal strain amplitude. The relation between fatigue life and the nominal strain amplitude, gained from the damage model, can satisfactorily characterize experimental data for a carbon-filled natural rubber.

Elasto-plasticity coupled with damage scope constructed chiefly for metal and polymers is extended to filled elastomers. The suggested simulation applies for static and likewise fatigue loadings. Expressed in the framework of thermodynamics and in 3D, it will take into account failures of construction subject to complex loadings to be calculated. The model brings in damage parameters $S, s$, and the critical damage $D_{c}$.

\section{Acknowledgement}

The author gratefully acknowledges the financial support of the presented research by the Grant Agency of the Czech Republic (project No. 103/09/1580).

\section{References}

[1] Lemaitre, J., Desmorat, R., Engineering Damage Mechanics: Ductile, Creep, Fatigue and Brittle Failures. Springer 2005.

[2] Brož P, Dobiáš D. Determining material parameters of damage. Acta Technica, 54, pp. 415-436, 2009.

[3] Desmorat, R., Unified framework for damage and fatigue of metals, concrete and elastomers. In XI International Conference on Fracture, Turin, Italy, 2005

[4] Desmorat, R., Cantournet, S., Thermodynamics modelling of internal friction and hysteresis of elastomers. In Second European Conference on Constitutive Models for Rubber, Vienne, Austria, 2001.

[5] Wang, B., Lu, H., Kim, G., A damage model for the fatigue life of elastomeric materials. Mechanics of Materials, 34, pp. 475-483, 2002.

[6] Lemaitre, J., Micro-mechanics of crack initiation. International Journal of Fracture, 42, pp. 87-99, 1990.

[7] Lu, H., Wang, B., Tan, G., Chen, W., Accelerated life prediction and testing of structural polymers under cyclic loading. In Long Term Durability of Structural Materials, pp. 195-205, 2001.

[8] Ogden, R.W., Non-linear Elastic Deformation. Ellis Horwood Limited, 1984.

[9] Twizell, E.H., Ogden, R.W., Non-linear optimization of the material constants in Ogden's stress-deformation function for incompressible isotropic elastomers. Journal of the Australian Mathematical Society, Series B 24, pp. 424-434, 1986. 